\title{
LECTURA Y ESCRITURA EN BIOLOGÍA. UNA EXPERIENCIA PEDAGÓGICA CON TIC
}

\section{Reading and Writing in Biology. A Pedagogical Experience with ICT}

\section{Leitura e escrita em biologia. Uma experiência pedagógica com TIC}

\section{Ana Reviglio*}

\section{Resumen}

El presente escrito describe una experiencia pedagógica en la cual la lectura y la escritura se integraron con nuevas tecnologías de la información y la comunicación (TIC) para funcionar como estrategias didácticas mediadoras del proceso de enseñanza y de aprendizaje de la biología. El trabajo se realizó desde la perspectiva teórica de la alfabetización académica (Carlino, 2013) con 32 alumnos de tercer año de una de las escuelas de Enseñanza Media de la Universidad Nacional de Rosario. Las actividades consistieron en lectura crítica de publicaciones científicas y de divulgación en formato impreso y digital, producción escrita de informes y de comentarios críticos y utilización de una red social para compartir información. Respecto de los resultados, en el desarrollo fue posible observar en los alumnos una serie de transformaciones actitudinales y cognitivas superadoras, que llaman a reflexionar sobre el rol que desempeñan la lectura y la escritura como recursos de aprendizaje de los sistemas de conceptos y métodos y de los usos del lenguaje específico de cada disciplina científica. Asimismo, las transformaciones impuestas por las tic demandan el desarrollo de competencias particulares relacionadas con las nuevas formas de leer, escribir y participar que encuentran en las prácticas áulicas un espacio apropiado para su aprendizaje.

Palabras clave: enseñanza de la biología; lectura; escritura; tecnologías de la información y la comunicación; aprendizaje

* Escuela Superior de Comercio Libertador General San Martín, Universidad Nacional de Rosario. Correo electrónico: evaluna73@gmail.com. 


\section{Abstract}

This paper shows the results of a pedagogical experience, which linked reading and writing skills to information and communication technologies (ICT) as didactic strategies to teach and learn biology. This work was based on academic literacy theory (Carlino, 2013) and was performed involving 32 third degree students of one of the Secondary Schools of the University of Rosario. The activities consisted of critical reading of specialized publications in printed and digital format, written production of reports and critical comments and use of a social network to share information. Regarding the results, during the experience, the authors could see positive transformations in both behavior and cognitive performance among pupils involved. This fact points to the importance of thinking deeply about the role of reading and writing as didactic strategies to learn specific language usages in each scientific field. Besides, ICT demands new skills to read, write, and participate in virtual spaces. At this point, schools are the most suitable place to learn about them.

Keywords: biology teaching; reading; writing; information and communication technologies; learning

\section{Resumo}

Este artigo descreve uma experiência pedagógica, na qual a leitura e a escrita foram integradas às novas tecnologias da informação e comunicação (TIC) para funcionar como estratégias educacionais mediando o processo de ensino e aprendizagem da biologia. 0 trabalho foi realizado sob a perspectiva teórica da alfabetização acadêmica (Carlino, 2013) com 32 alunos do terceiro ano de uma das escolas secundárias da Universidade Nacional de Rosário. As atividades consistiram na leitura crítica de publicações científicas e de divulgação em formato impresso e digital, produção escrita de relatórios e comentários críticos e uso de uma rede social para compartilhar informações. Quanto aos resultados, no desenvolvimento foi possível observar nos alunos uma série de superações de transformações atitudinais e cognitivas, que exigem refletir sobre o papel da leitura e da escrita como recursos de aprendizagem dos sistemas de conceitos e métodos e de os usos específicos da linguagem de cada disciplina científica. Da mesma forma, as transformações impostas pelas TIcs exigem o desenvolvimento de competências específicas relacionadas às novas formas de ler, escrever e participar que encontram nas práticas em sala de aula um espaço adequado para sua aprendizagem.

Palavras chave: ensino de biologia; leitura; escrita; tecnologias da informação e comunicação; aprendizado 


\section{Introducción}

El entramado complejo que caracteriza a nuestra sociedad actual demanda que nuestros estudiantes sean formados como ciudadanos responsables, solidarios, reflexivos, respetuosos del valor de la libertad y capacitados para intervenir sobre la realidad y transformarla. En este sentido, las habilidades relacionadas con la comunicación, el trabajo colaborativo y la flexibilidad para adaptarse a contextos diversos y cambiantes se vuelven recursos ${ }^{1}$ valiosos para la inclusión y la participación.

Dado que no se puede pensar la comunicación prescindiendo del lenguaje, el desarrollo de competencias lingüísticas se plantea como requisito indispensable para establecer comunicaciones efectivas en los diferentes contextos de interacción social, afectiva, cultural, laboral y académica.

El entrenamiento en el hábito de la lectura y la escritura es una herramienta válida para enriquecer el lenguaje y desarrollar capacidades que favorezcan la comunicación (comprensión, síntesis, expresión, abstracción, secuenciación lógica, opinión). Del mismo modo, las transformaciones propuestas por las nuevas tecnologías de la información y la comunicación (TIC) demandan el desarrollo de competencias que permitan formar parte de un nuevo espacio social virtual de participación, colaboración, inclusión, integración, experimentación y conexión de actores. Frente a estas consideraciones, surge esta propuesta de enseñanza en la cual la lectura y la escritura integradas con recursos TIC se proponen como estrategias didácticas mediadoras del proceso de aprendizaje.

Esta experiencia se llevó a cabo en una división de tercer año del turno de la mañana de la Escuela Superior de Comercio Libertador General San Martín, cuyas características se describen en el apartado "Diseño".

\section{Justificación de la propuesta}

Bogel y Hjorthoj (citados en Carlino et ál., 2013) afirman que una disciplina es un espacio discursivo y retórico tanto como conceptual. De esta manera, su aprendizaje implica apropiarse de sus sistemas de conceptos y métodos, y de los usos del lenguaje específico. Estos últimos reconocen una naturaleza práctica y situada, se deben aprender dentro del contexto en el cual se producen y son mediados por aquellos actores que tienen experiencia en su utilización.

1 En este contexto, recurso hace referencia a todo elemento que el docente emplea en el diseño o desarrollo del currículo (por su parte o la de los alumnos) para aproximar o facilitar los contenidos, mediar en las experiencias de aprendizaje, desarrollar habilidades cognitivas, o facilitar o enriquecer la evaluación (Blázquez y Lucero, 2002).
Así, se considera que en cada área de conocimiento se lee y se escribe de formas particulares.

Desde esa perspectiva, la lectura y la escritura no son habilidades generales que una vez aprendidas se pueden aplicar a cualquier texto situado en cualquier contexto. Fundamentalmente, aquellos aspectos que se vinculan con el contenido y con el propósito de la escritura se reconocen como particulares de contextos específicos o de ciertas comunidades de práctica. De esta manera, los elementos del discurso escrito que se relacionan con el significado (desarrollo conceptual, adecuación a la situación comunicativa, argumentación válida, jerarquización de conceptos) no son aceptados de la misma manera en los ámbitos de las diferentes disciplinas. Esto determina que para aproximarse a los modos de lectura y escritura propios de cada área de estudio no basta la simple extensión de los procedimientos de alfabetización básicos, sino que es necesario reconstruir esas habilidades para transformarlas en herramientas que permitan intervenir en contextos disciplinares específicos. Así, tanto la lectura como la escritura dejan de reconocer una condición autónoma, independiente o separada del contenido que se lee o que se escribe.

En los contextos escolares, los docentes podemos pensar la escritura de dos formas diferentes: como un conocimiento previamente adquirido por los alumnos que se utiliza para comunicar una idea o un pensamiento preexistente, o como un objeto de enseñanza, un contenido curricular que se debe abordar específicamente en el desarrollo de la asignatura. Esta segunda mirada propone que la escritura es una actividad que permite reflexionar sobre lo que se escribe, modificando así el proceso cognitivo y aportando a la construcción de ideas nuevas, superadoras, que no existían antes de comenzar a escribir. En este aspecto, la producción de textos escritos se transforma en una estrategia didáctica eficiente para mediar la construcción de conocimientos propios de las distintas disciplinas.

Con relación a la lectura, es frecuente proponerla como una competencia que se desarrolla independientemente del contenido del material que se lee. Así, se da por sentado que aquellos alumnos que hayan aprendido a leer de forma general cuentan con los recursos cognitivos necesarios para leer bibliografía específica de cualquier asignatura y son capaces de acceder al significado de los textos por simple decodificación. A la luz de esta postura, la mediación de la lectura por parte del docente no resultaría necesaria porque un mismo texto solo admite una única comprensión posible. Sin embargo, el proceso de lectura también se puede abordar a partir de un modelo interactivo en el cual el significado de lo leído se construye combinando elementos del texto y elementos del lector, 
en especial sus conocimientos previos y su propósito de lectura. De este modo, los recursos que utiliza un alumno para aproximarse a un texto condicionan las interpretaciones, las jerarquizaciones y las relaciones sustantivas que podrá establecer. En consonancia, la intervención del docente conocedor del marco conceptual de su disciplina resulta fundamental para orientar a los estudiantes en la búsqueda de aquella información que resulta significativa para cada contexto. Asimismo, la mediación docente ${ }^{2}$ también cobra relevancia a la hora de confrontar las diversas interpretaciones de la lectura con el estado del conocimiento disciplinar.

Lo expuesto justifica que las formas de leer y de escribir en cada disciplina formen parte de los contenidos por enseñar. Así, los alumnos podrán desarrollar competencias de interpretación y producción de textos que les permitan apropiarse de los saberes específicos.

En el mismo sentido, la lectura y la escritura se constituyen en herramientas de estudio o estrategias de aprendizaje. Y como los modos de estudiar dependen del contenido que se va a abordar, la mediación docente resulta de particular importancia a fin de guiar la selección y jerarquización de la información. El desarrollo de estas competencias se verá reflejado en la eficacia con la que los alumnos tomen apuntes en clase, elaboren resúmenes o validen información disponible en internet.

Es importante destacar que la lectura y la escritura se pueden considerar estrategias de aprendizaje solo cuando se reconocen como procesos intelectuales que estimulan la construcción de relaciones sustantivas entre los conocimientos previos y el nuevo saber. Por lo tanto, no cualquier tarea referida a la producción o a la comprensión de textos escritos realizada en contextos áulicos es igualmente eficiente como actividad cognitiva. Del mismo modo, es interesante resaltar que la lectura y la escritura disciplinares son habilidades que demandan tiempo y que requieren secuencias didácticas continuas ya que se adquieren a través de la experiencia mediada.

Así, es posible concluir que para favorecer la apropiación del conocimiento disciplinar se deben entender las prácticas de lectura, de escritura y de estudio como contenidos curriculares por enseñar en las diferentes asignaturas. Integrar actividades de lectura y escritura a la práctica áulica puede dar lugar a intercambios más participativos,

2 La postura del docente como mediador implica entenderlo como promotor del aprendizaje significativo a partir de espacios de reflexión y del uso de estrategias que permitan la construcción del conocimiento, además de desarrollar habilidades de pensamiento vinculadas a la epistemología de la disciplina (Parra, 2014). en los cuales dificultades habituales durante el proceso de aprendizaje, como "interpretar información de manera inapropiada para el contexto", "no expresar ideas o relaciones de forma pertinente", o "utilizar terminología que no pertenece a una determinada comunidad de práctica", se transformen en estrategias didácticas que lo faciliten.

En consecuencia, en esta propuesta, la lectura y la escritura se conciben como instrumentos de aprendizaje específicos e inacabados que se trabajan y se orientan de acuerdo con el contenido y el propósito de lo escrito. Este trabajo se fundamenta en una construcción teórica de la escritura que la entiende como una actividad social, lingüística y cognitiva que permite repensar aquello sobre lo que se escribe y dar lugar a ideas que no existían antes de escribir. Entonces, la escritura se transforma en un instrumento de elaboración de ideas. De igual manera, la construcción teórica de la lectura se apoya en los modelos interactivos y constructivos que consideran que el significado de lo leído no solo depende de lo que aporta el texto, sino también de lo que aporta el lector con relación a sus conocimientos previos y a su propósito de lectura. Así, las categorías conceptuales que despliega un alumno para interactuar con un texto van a permitir que reconozca ciertas ideas y que no identifique otras interpretaciones potenciales. Estas categorías conceptuales son las que intervienen también para discriminar entre ideas centrales e ideas secundarias. La intervención docente, a partir del conocimiento del marco conceptual disciplinar y de la experiencia lectora, orienta para saber qué buscar en los textos a fin de identificar la información relevante.

\section{Diseño}

A partir de la integración de las premisas expuestas, se elaboró una propuesta de enseñanza en la cual se articularon actividades diversas, que incluyen la lectura crítica de publicaciones científicas y de divulgación en formato impreso y digital, la producción escrita de informes y de comentarios críticos y la utilización de una red social para compartir información. De esta manera, se esperó poder reconstruir las habilidades de lectura y escritura para transformarlas en herramientas que permitan producir y apropiarse del conocimiento en un contexto dominado por prácticas, soportes y géneros discursivos novedosos. ${ }^{3}$

La experiencia se desarrolló durante el ciclo lectivo 2014 en la Escuela Superior de Comercio Libertador General San Martín, una de las tres escuelas de Enseñanza Media de la Universidad Nacional de Rosario, provincia de Santa

3 En este contexto, el término novedoso hace referencia a los cambios impuestos por la textualidad electrónica en relación con la impresa para la percepción de la cultura escrita (Chartier, 2014). 
Fe, República Argentina. La escuela está ubicada en el macrocentro de la ciudad y tiene una población estudiantil de 1200 alumnos, distribuidos en 8 divisiones (4 en turno mañana y 4 en turno tarde) de cada uno de los 5 años que conforman el nivel medio. Los alumnos acceden a la escuela luego de aprobar un examen de ingreso que evalúa contenidos de matemática y lengua española. La enseñanza en la escuela es pública y gratuita (con la posibilidad de que los alumnos que lo requieran accedan a becas de estudio), y la población estudiantil proviene de sectores socioeconómicos y culturales diversos, lo que le otorga un grado significativo de heterogeneidad. La propuesta que se presenta involucró a los alumnos de una división de tercer año del turno de la mañana, conformada por 20 mujeres y 12 varones de entre 14 y 16 años de edad. Las actividades se realizaron en grupos de 4 alumnos (conformados voluntariamente) para favorecer la construcción colectiva y colaborativa.

\section{Objetivos}

Se planteó que a partir del desarrollo de la propuesta "Lectura y escritura en biología" los alumnos serían capaces de:

- Analizar de manera crítica bibliografía correspondiente a la biología (incluyendo publicaciones científicas, de divulgación y libros de texto).

- Reelaborar de forma escrita los mensajes comunicados por la bibliografía desde una perspectiva personal, utilizando argumentos y estrategias que fundamenten la construcción de sentido del mensaje.

- Confrontar la información analizada con datos del contexto.

- Expresar opiniones y conclusiones utilizando lenguaje propio del área de la biología.

- Promover el aprendizaje significativo y colaborativo y la aplicación de las nuevas tic.

\section{Desarrollo}

La propuesta se presentó al comienzo del ciclo lectivo (marzo de 2014). A partir de la lectura crítica y reflexiva de un texto asignado indistintamente en formato impreso o digital, cada grupo redactó un documento escrito en el que se que reelaboró la información recibida incluyendo una conclusión o relación significativa con fenómenos de la vida cotidiana. Las producciones se publicaron en un soporte digital creado para la actividad (https://www. facebook.com/groups/757673317597872/).
Asimismo, cada grupo elaboró un comentario en el que expresaba su opinión sobre una de las publicaciones de sus compañeros, que también se publicó en el soporte digital.

Cada secuencia didáctica tuvo una duración de tres semanas y se repitió tres veces a lo largo del ciclo lectivo.

La primera actividad se llevó a cabo con base en el libro Una tumba para los Romanov y otras historias con ADN (Alzogaray, 2008). En segundo lugar, se trabajó sobre el libro Cerebro y memoria (Goio, et ál., 2012). Finalmente, la tercera actividad se basó en Los remedios de la abuela: mitos y verdades de la medicina casera (Edelzstein, 2012).

\section{Criterios de evaluación}

Para evaluar las competencias y los aprendizajes desarrollados se tuvieron en cuenta los siguientes criterios, mensurados a través de una matriz de valoración que se presenta en la tabla 1:

- creación de productos originales como medio de expresión personal y grupal.

- utilización pertinente del lenguaje propio del área de la biología (tanto en lo referido al vocabulario como en la adecuación al Nivel Secundario de Enseñanza).

- responsabilidad individual y grupal en el aprendizaje.

- transferencia de conocimientos al aprendizaje de nuevas tecnologías. 
Tabla 1. Matriz de valoración para la evaluación

\begin{tabular}{|c|c|c|c|c|}
\hline & Excelente & Muy buena & Buena & En proceso \\
\hline $\begin{array}{l}\text { Redacción general } \\
\text { (coherencia, cohesión y } \\
\text { ortografía) }\end{array}$ & $\begin{array}{l}\text { Se observa una cuidadosa } \\
\text { selección en la secuencia } \\
\text { de párrafos. No presenta } \\
\text { ningún error ortográfico. } \\
\text { Se lee con fluidez. }\end{array}$ & $\begin{array}{l}\text { El texto se organiza de } \\
\text { manera coherente y } \\
\text { cumple con su propósito. } \\
\text { Presenta solo algunos } \\
\text { errores ortográficos. Es } \\
\text { claro y se comprende sin } \\
\text { dificultad. }\end{array}$ & $\begin{array}{l}\text { Se intenta separar el texto } \\
\text { por ideas sin conseguir } \\
\text { darle coherencia y } \\
\text { cohesión. Algunas áreas } \\
\text { del texto son claras y otras } \\
\text { carecen de claridad y son } \\
\text { difíciles de comprender. } \\
\text { Presenta mínimos errores } \\
\text { ortográficos. }\end{array}$ & $\begin{array}{l}\text { El texto es un continuo en } \\
\text { donde no se separan las } \\
\text { ideas. Los párrafos son } \\
\text { incompletos y difíciles } \\
\text { de comprender. Presenta } \\
\text { gran cantidad de errores } \\
\text { ortográficos. }\end{array}$ \\
\hline Reflexiones personales & $\begin{array}{l}\text { Es coherente, claro } \\
\text { y preciso. Utiliza } \\
\text { variedad de argumentos } \\
\text { y estrategias para } \\
\text { fundamentar su reflexión. }\end{array}$ & $\begin{array}{l}\text { Es coherente, claro } \\
\text { y preciso. Utiliza } \\
\text { argumentos para } \\
\text { fundamentar su reflexión. }\end{array}$ & $\begin{array}{l}\text { Se establece una } \\
\text { idea central, pero los } \\
\text { argumentos no se } \\
\text { exponen con claridad. No } \\
\text { utiliza estrategias. }\end{array}$ & $\begin{array}{l}\text { No se establece una idea } \\
\text { central que sintetice la } \\
\text { reflexión. No se presentan } \\
\text { argumentos que validen } \\
\text { la reflexión. }\end{array}$ \\
\hline Vocabulario específico & $\begin{array}{l}\text { El texto se elabora con } \\
\text { una alta pertinencia de } \\
\text { vocabulario específico } \\
\text { de la disciplina y es } \\
\text { adecuado al nivel } \\
\text { secundario. }\end{array}$ & $\begin{array}{l}\text { El texto se elabora con } \\
\text { vocabulario específico de } \\
\text { la actividad y es adecuado } \\
\text { al nivel secundario. }\end{array}$ & $\begin{array}{l}\text { Solo algunos fragmentos } \\
\text { del texto presentan } \\
\text { vocabulario específico, } \\
\text { pero no lo hacen de } \\
\text { manera ordenada y } \\
\text { comprensible. }\end{array}$ & $\begin{array}{l}\text { No se domina el } \\
\text { vocabulario específico de } \\
\text { la temática. }\end{array}$ \\
\hline $\begin{array}{l}\text { Mejoras y rediseños de las } \\
\text { producciones }\end{array}$ & $\begin{array}{l}\text { Se presentan borradores } \\
\text { de las producciones } \\
\text { permanentemente. Los } \\
\text { trabajos se reelaboran } \\
\text { constantemente para } \\
\text { lograr producciones de } \\
\text { alta calidad. }\end{array}$ & $\begin{array}{l}\text { Se presentan borradores } \\
\text { permanentemente. La } \\
\text { reelaboración de los } \\
\text { trabajos se adecúa al } \\
\text { objetivo de la actividad. }\end{array}$ & $\begin{array}{l}\text { No siempre se presentan } \\
\text { borradores de los } \\
\text { trabajos. La reelaboración } \\
\text { no siempre contempla } \\
\text { mejoras en las } \\
\text { producciones. }\end{array}$ & $\begin{array}{l}\text { Casi nunca o nunca se } \\
\text { presentan borradores de } \\
\text { las producciones. No se } \\
\text { observa reelaboración de } \\
\text { los trabajos. }\end{array}$ \\
\hline $\begin{array}{l}\text { Adecuación al calendario } \\
\text { de trabajo }\end{array}$ & $\begin{array}{l}\text { Siempre cumple con las } \\
\text { fechas de entrega de } \\
\text { trabajos. }\end{array}$ & & & $\begin{array}{l}\text { No se cumplen las fechas } \\
\text { pautadas de entrega de } \\
\text { trabajos. }\end{array}$ \\
\hline $\begin{array}{l}\text { Participación en los } \\
\text { comentarios }\end{array}$ & $\begin{array}{l}\text { Siempre realiza } \\
\text { comentarios en } \\
\text { los trabajos de los } \\
\text { compañeros. }\end{array}$ & & & $\begin{array}{l}\text { No realiza comentarios en } \\
\text { las producciones de los } \\
\text { compañeros. }\end{array}$ \\
\hline $\begin{array}{l}\text { Creatividad en las } \\
\text { presentación de la } \\
\text { información } \\
\text { (utilización de imágenes, } \\
\text { hipervínculos, } \\
\text { animaciones) }\end{array}$ & $\begin{array}{l}\text { Diseño original para } \\
\text { presentar la información. } \\
\text { Se utilizan recursos } \\
\text { buenos y adecuados. }\end{array}$ & & $\begin{array}{l}\text { Buen diseño de las } \\
\text { presentaciones. Utiliza } \\
\text { pocos recursos para } \\
\text { enriquecerlas. }\end{array}$ & $\begin{array}{l}\text { La secuencia con que se } \\
\text { presenta la información } \\
\text { es confusa o se relaciona } \\
\text { poco con la temática } \\
\text { investigada. No utiliza } \\
\text { recursos. }\end{array}$ \\
\hline
\end{tabular}




\section{¿Qué fue lo que dejó la experiencia?}

Con relación a los aportes a la práctica docente del proyecto "Lectura y escritura en biología" existen ciertos aspectos que merecen ser destacados.

- Resistencia a lo novedoso. Dentro del conjunto de actitudes observadas en los alumnos durante el desarrollo de esta propuesta resultó llamativo el rechazo sistemático ante la aplicación de estrategias didácticas no tradicionales y el uso de herramientas TIC para producir y compartir información. El diálogo permitió descubrir que estas actitudes se debían a una falta de conocimientos para entender la lógica de funcionamiento de los recursos digitales o su aplicación para realizar tareas fuera de lo meramente lúdico o social. En la planificación de actividades, el discurso de los nativos digitales tuvo una influencia excesiva, ya que se dio por sentado que los alumnos contaban con una serie de habilidades que, en realidad, no tenían. La gran mayoría demostró carecer de estrategias para utilizar redes sociales, para buscar y validar información de forma crítica, para comprender el funcionamiento de los buscadores y de las herramientas de aprendizaje.

- Proceso de escritura superador. La producción de textos escritos como estrategia didáctica para mediar la construcción de conocimientos disciplinares habilitó espacios de reflexión sobre lo escrito, lo que permitió arribar a producciones superadoras. En este punto, la presentación de borradores desempeñó un papel destacado. A partir de las intervenciones docentes, los alumnos pudieron complejizar en forma creciente los desarrollos conceptuales, la adecuación a la situación comunicativa, la argumentación y la jerarquización de ideas hasta lograr mayor coherencia, cohesión y ortografía.

- Integración de diversos lenguajes comunicativos. Las consignas que propusieron integrar textos e imágenes para lograr un objetivo concreto posibilitaron a los alumnos explorar distintas modalidades de expresión, diversificar el concepto de comunicación e integrar recursos alternativos para modificar el impacto comunicativo.

- Trabajo colaborativo. Una de las destrezas requeridas en el contexto actual de la sociedad de la información y la comunicación es el trabajo colaborativo, que pone énfasis en la interacción y la construcción colectiva de conocimientos. Resolver las actividades pautadas de forma grupal y habilitar espacios de participación colectiva para comentar críticamente las producciones de otros permitió reflexionar sobre las ideas propias, compartir saberes, interpretar situaciones desde distintas perspectivas, estimular la creatividad, arribar a acuerdos, compartir responsabilidades y reconocer fortalezas y debilidades. Algunos grupos tuvieron dificultades significativas para producir de forma colaborativa, lo que los motivó a ejercer de manera responsable el proceso de toma de decisiones para poder avanzar en la propuesta.

- Desconcierto ante la autonomía. Otro aspecto relevante es la resistencia inicial de los alumnos a autogestionar su aprendizaje. El desconcierto ante lo novedoso, el temor a lo desconocido, la baja tolerancia a la frustración que caracterizan a este grupo etario, sumados a la dificultad para el ejercicio de la autonomía y la toma de decisiones provocaron cierto estado de tensión al comenzar el trabajo. A medida que los estudiantes se fueron familiarizando con la resolución de las actividades y pudieron realizar procesos metacognitivos para reflexionar sobre sus propios aprendizajes, el clima áulico favoreció la implementación de la propuesta. En este sentido, la integración de recursos TIC representó una alternativa eficiente para estimular la metacognición. Al ofrecer la posibilidad de implementar distintos modos de produciry compartir información, las TIC permitieron elegir las estrategias más eficientes para un contexto determinado.

- Implicancias en los procesos de enseñanza y aprendizaje de la biología. La construcción de aprendizajes en biología requiere que los estudiantes desarrollen habilidades de pensamiento que contribuyan a integrar estructuras, procesos y efectos que, apartándose de las imágenes cotidianas del mundo macroscópico conocido, posibiliten la comprensión de fenómenos y la toma de decisiones asertivas. Estas habilidades incluyen observación, identificación, diferenciación, ordenamiento, organización, descripción, crítica, opinión, comparación, inferencia y jerarquización. El proceso de lectura que permite acceder al sentido y significado de los textos favorece el desarrollo de estas habilidades ya que requiere el análisis de la organización, el ordenamiento y las relaciones de las ideas expuestas.

- A medida que avanza en la lectura, el lector puede advertir que en el texto hay mucha más información que la que se menciona expresamente y que es él quien la va haciendo explícita a partir de desarrollar habilidades de inferencia (Pellicer, 2015). 
La valoración del título del texto, del contexto donde aparece, el reconocimiento de ideas principales y secundarias (mediante técnicas de subrayado o resaltado), la identificación de las formas en las que el autor comienza, continúa y concluye esas ideas y cómo las enlaza entre sí permiten desarrollar habilidades de pensamiento que les posibilitarán a los estudiantes asumir un rol activo en la construcción de nuevos aprendizajes en biología. Del mismo modo, el proceso de escritura implica hacer uso de recursos lingüísticos que establezcan las relaciones que le dan coherencia y cohesión a un texto (y por lo tanto, a la comunicación de un mensaje), identificando cuál es el punto de partida de una acción, cuáles son sus resultados, qué condiciones de contexto acompañan y cuál es el orden temporal en que se producen (en secuencia o en simultáneo).

La posibilidad de utilizar estos recursos de forma pertinente favorece la construcción de conocimientos en biología a partir del desarrollo de secuencias de pensamiento válidas para la aproximación a la lógica de los contenidos de la disciplina. Otro aspecto significativo del uso recursos de lectura y escritura para la enseñanza y el aprendizaje de la biología consiste en la posibilidad de pensar estrategias de evaluación que tengan en cuenta la distancia que hay entre el sentido que reconstruyó el lector y el que el autor quiso darle al texto. En ese sentido, tanto la escritura (y reescritura) de borradores como la escritura de comentarios de opinión sobre las producciones de otros grupos funcionaron como insumos para la evaluación del proceso, lo que permitió incrementar de manera progresiva la complejidad de los aprendizajes logrados.

- El rol de las TIC en los procesos de enseñanza y aprendizaje de la biología. El contexto actual nos posiciona en la sociedad de la información y el conocimiento (Crovi Druetta, 2004), conformada como un medio vasto y flexible a partir de la irrupción de las TIC, que representan una innovación en las formas de producir y circular conocimiento. Esto ha dado como resultado la conformación de un nuevo espacio social virtual de producción de información, de participación, colaboración, inclusión, integración y conexión de actores que demanda el desarrollo de nuevas competencias para poder formar parte. Esta realidad hace necesario reorganizar las prácticas de enseñanza y de aprendizaje de acuerdo a estas nuevas formas de producir saberes que incluyen la hipertextualidad, la conectividad, la colectividad y la interactividad. Accionar de modo crítico, creativo, reflexivo y responsable sobre la información disponible para aplicarla de forma eficiente a diversos contextos y entornos de aprendizaje y para construir conocimiento significativo a partir de ella demanda el desarrollo de competencias y de herramientas cognitivas pertinentes; las instituciones educativas desempeñan un rol central como mediadoras de ese proceso.

En la experiencia relatada, los estudiantes desarrollaron habilidades para el uso de herramientas digitales (redacción y lectura de documentos; búsqueda, selección y utilización de imágenes y recursos multimedia; interacción a través de una red social) que promovieron formas novedosas de aproximarse al conocimiento, más adecuadas para favorecer el desarrollo de competencias digitales. Asimismo, en algunas ocasiones, los tiempos de trabajo áulico previstos no fueron suficientes para lograr los desempeños esperados, y la calidad de la conectividad no siempre permitió el acceso eficiente a los recursos tecnológicos. Estas situaciones ofrecieron la posibilidad de extender el aprendizaje fuera del espacio áulico y presentarles a los alumnos dos nuevas propuestas para construir conocimientos: el aprendizaje ubicuo (Burbules, 2009) y el aula aumentada (Sagol, 2012).

Ambos conceptos ofrecen formas menos compartimentadas de concebir la enseñanza y el aprendizaje (tanto en tiempos como en espacios) y favorecen la integración del aprendizaje áulico con aquellos que se producen en otros ámbitos de interacción. La escuela y la clase presencial ya no son las únicas instancias para aprender, sino que cualquier momento y cualquier lugar se proponen como posibilidades viables para que este ocurra. La presencia del docente puede extenderse por fuera del calendario académico y de esa manera ofrecer nuevos espacios de interacción e intercambio. En este aspecto, las Tıc habilitan posibilidades que no podrían pensarse si se dejan de lado en la planificación de la secuencia didáctica. La autonomía que se va generando con estas nuevas formas de aprender funciona como un estímulo eficiente para el ejercicio de la metacognición. Al interactuar con recursos didácticos variados que combinan lógicas diferentes, los alumnos pueden reconocer y seleccionar aquellos que resultan más apropiados para la construcción de nuevos saberes. 


\section{Consideraciones finales}

Este trabajo se fundamenta en la concepción de que leer y escribir son prácticas inherentes al proceso de aprendizaje y que, como tales, funcionan como actividades potentes y centrales para elaborar el conocimiento implicado. La implementación de esta propuesta de enseñanza mostró que los espacios de reflexión sobre lo escrito que habilita la escritura permiten a los alumnos elaborar producciones superadoras que favorecen la progresiva complejización de los aprendizajes y reafirman la concepción de la lectura y la escritura como procesos intelectuales y como instrumentos para pensar y establecer relaciones. Del mismo modo, el proceso de lectura que permite acceder al sentido y significado de los textos favorece el desarrollo de las habilidades de pensamiento que se ponen en juego a la hora de construir aprendizajes en biología.

En otro orden de ideas, la integración de las TIC a la propuesta de enseñanza posibilitó el desarrollo de competencias y habilidades cognitivas necesarias para que los alumnos puedan formar parte del nuevo espacio social virtual de producción de saberes, de participación, colaboración, inclusión, integración y conexión de actores que quedó inaugurado a partir de la irrupción masiva de las nuevas tecnologías en los espacios académicos, laborales y de interacción social.

Los aportes de este trabajo también invitan a reflexionar acerca de la práctica docente: sobre las concepciones de lectura y escritura que sostenemos, sobre cuán inacabados consideramos que son sus procesos de aprendizaje y sobre nuestro rol heterorregulador en la producción e interpretación de textos que resultan novedosos para nuestros alumnos porque actualizan los contenidos que se van a enseñar. Esto va a permitir identificar si la lectura y la escritura están presentes en nuestra práctica de forma periférica, o si se integran para trabajar los contenidos disciplinares acompañadas por la intervención docente.

En la misma línea de reflexión, y de acuerdo con lo observado durante el desarrollo de la propuesta, es importante destacar que la integración de nuevas tecnologías implica reconocer la falta de competencias que tienen los alumnos para reconocer la lógica de funcionamiento de ciertos recursos tecnológicos. Este hecho invita a reflexionar sobre el alcance que tiene la expresión nativos digitales, que da cuenta de la familiaridad y las destrezas que este grupo etáreo despliega a la hora de hacer un uso lúdico y recreativo de los recursos tecnológicos, pero invisibiliza su ausencia de competencias para integrar nuevas tecnologías a los campos del aprendizaje y del trabajo. Esta situación demanda igualmente un rol docente hete- rorregulador para que las tecnologías se transformen en recursos mediadores eficientes del aprendizaje.

La propuesta de enseñanza "Lectura y escritura en biología" se diseñó con el propósito de promover una interacción más eficiente entre la lectura, la escritura y las nuevas tecnologías en contextos escolares de aprendizaje. Asimismo, representa un intento de revalorizar estos dos procesos como herramientas para la construcción de la subjetividad, la inclusión social, la apropiación de saberes considerados valiosos y la formación de competencias. En el escenario actual, las nuevas tecnologías imparten características particulares a la cultura de lo escrito, que es imperativo tener en cuenta a la hora de analizar su transmisión en los espacios escolares.

\section{Referencias}

Alzogaray, R. (2008). Una tumba para los Romanov y otras historias con ADN. Buenos Aires: Siglo XXI.

Archanco, P. (2015).. Sobre la práctica de la lectura en la escuela: supuestos, continuidades y rupturas. Flacso.

Blázquez, F. y Lucero, M. (2002). Los medios y recursos en el proceso didáctico. En A. Medina y F. Mata. Didáctica general. Madrid, España: Pearson Educación.

Burbules, N. (2009), Meanings of "ubiquitous learning". Exploring the anywhere/anytime possibilities for learning in the age of digital media. Champaign, IL, University of Illinois Press.

Carlino, P., Iglesia, P., Bottinelli, L., Cartolari, M., Laxalt, I. y Marucco, M. (2013). Leer y escribir para aprender en las diversas carreras y asignaturas de los IFD que forman a profesores de enseñanza media: concepciones y prácticas declaradas de los formadores de docentes. Buenos Aires: Ministerio de Educación de la Nación. http://red.infd.edu.ar/articulos/nuevo-estudio-nacional-leer-y-escribir-para-aprender/.

Chartier, R. (2014). ¿Es que el libro ha muerto? Reflexiones en torno a las nuevas textualidades. En Diploma Superior en Lectura, Escritura y Educación-Flacso, campus virtual.

Crovi Druetta, D. (2004). Sociedad de la información y el conocimiento. Entre lo falaz y lo posible. Buenos Aires: La Crujía.

Edelzstein (2012). Los remedios de la abuela: mitos y verdades de la medicina casera. Buenos Aires: Siglo xxI. 
Goio, M. G., Federman, N., Navarro, N. A., Cuestas, V. M. y Würschmidt, A. E. (2012). Cerebro y memoria. Buenos Aires: Ministerio de Educación de la Nación.

Parra, K. (2014). El docente y el uso de la mediación en los procesos de enseñanza y aprendizaje. Revista de Investigación [en línea]. http://www.redalyc. org/articulo.oa?id=376140398009.

Pellicer, A. (2015). La comprensión lectora del texto expositivo-informativo. http://www.sems.gob.mx/ work/models/sems/Resource/12180/1/images/ comprension_informativo.pdf.
Sagol, C. (2012). El aula aumentada. En Seminario Web 2012: "Aprendizaje ubicuo y modelos 1 a 1 ". IIPE-Unesco y Flacso-Argentina, 14 al 16 de marzo. http://www.webinar.org.ar/conferencias/aprendizaje-ubicuo-modelos-1-1-experiencias-propuestas-del-portal-educar.

Uribe Tirado, A. (2011). Competencias informáticas e informacionales. http://ci2-colombia.blogspot. com.ar/. 